\title{
Palliative Embolisation of Brain
} Arteriovenous Malformations Presenting
with Progressive Neurological Deficit

\author{
M. AL-YAMANY, K. G. TERBRUGGE, R. WILLINSKY, W. MONTANERA, \\ M. TYMIANSKI, M.C. WALLACE
}

Radiology and Surgery, University of Toronto, Head Division of Interventional and Diagnostic Neuroradiology, The Western Hospital, University Health Network, Toronto, Ontario, Canada

Key words: palliative embolisation, progressive neurological deficit, brain arteriovenous malformations

\section{Summary}

Large arteriovenous malformations (AVMs) located in eloquent areas of the brain are generally considered incurable because of the high morbidity and mortality associated with their treatment. When these patients develop a progressive neurological deficit they in time often become severely disabled. This report presents the results of palliative embolisation in this subgroup of patients.

Analysis of our data-base of 714 patients with known brain $A V M$ s revealed 17 patients who presented with progressive neurological deficit and who underwent palliative embolisation as the therapeutic modality of choice for management of their AVM. One patient was excluded due to lack of follow-up and two were excluded because they later received radiation therapy. Following embolisation $43 \%$ had improvement of their neurological deficit, 50\% stabilized and $7 \%$ continued to deteriorate and these clinical results persisted for an average of more than 2 years follow-up.

Transient neurological morbidity associated with embolisation treatment was $7 \%$ and there was no permanent morbidity and no mortality.
Palliative embolisation of brain AVMs presenting with progressive neurological deficits arrested deterioration in more than $90 \%$ of patients and was associated with low morbidity and no mortality.

\section{Introduction}

Moderate and large brain arteriovenous malformations in eloquent locations are difficult to treat by means of surgery ${ }^{1,2,3}$. The risk associated with surgical resection in most of these cases exceeds the morbidity and mortality linked with the natural history ${ }^{4,5}$. Embolisation to reduce the size of the AVM to make it suitable for radiosurgery is often not achievable, and many of these patients are left untreated. If these patients present with a progressive neurological deficit, then few treatment options remain.

Our multidisciplinary team has recommended partial embolisation in an attempt to stop the progression of the neurological deficit. We present our review of patients in whom we used this treatment option. 

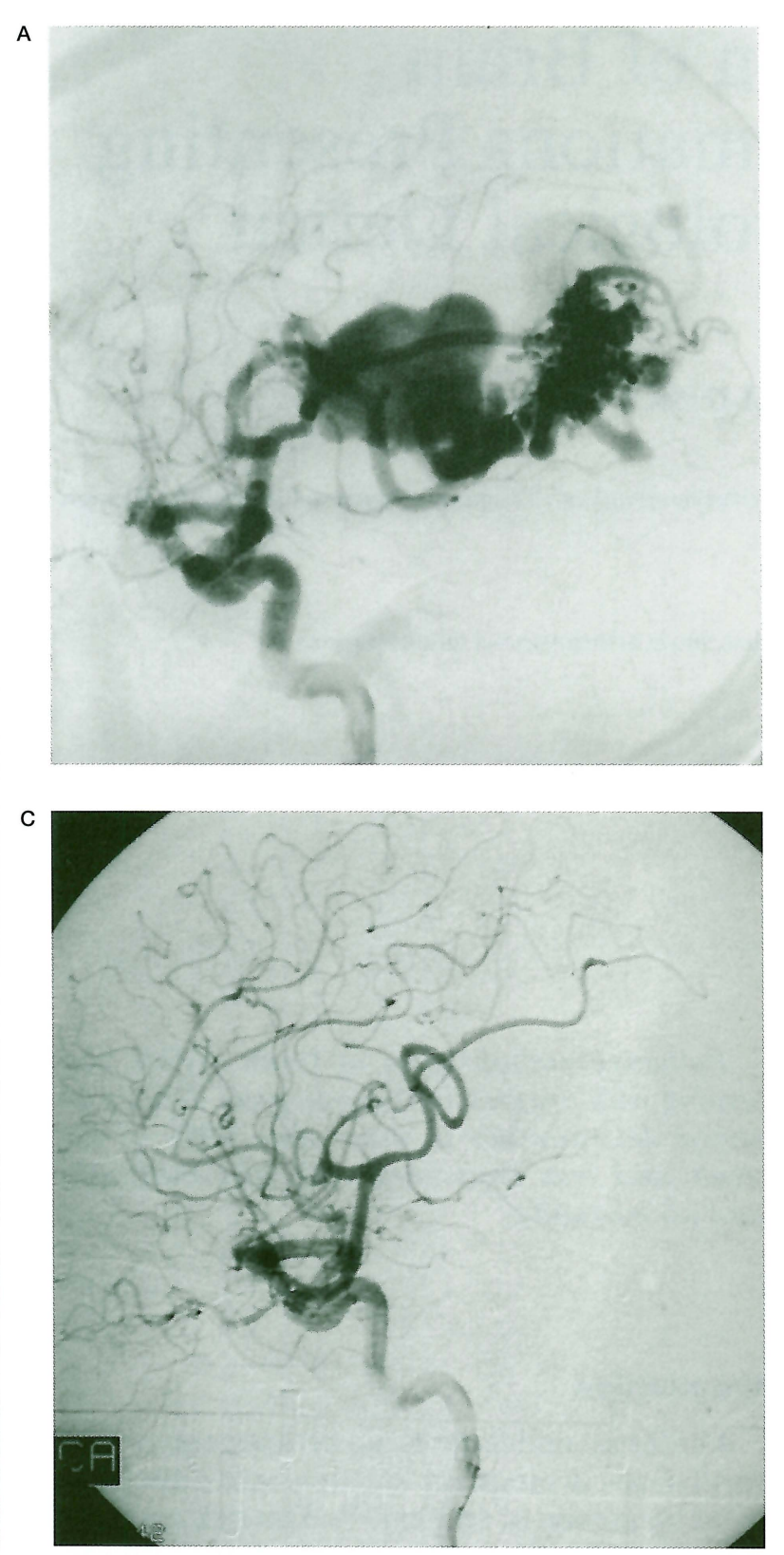

\section{Material and Methods}

We reviewed the data from 714 patients with brain arteriovenous malformations. The data were collected prospectively from 1989 and retrospectively between 1984 and 1989. 24 patients were referred to our clinic with a progressive neurological deficit and all had a Spetzler and Martin grade IV or V brain $\mathrm{AVM}^{6}$. $\mathrm{Pa}$ tients with Vein of Galen malformation were excluded from this study.

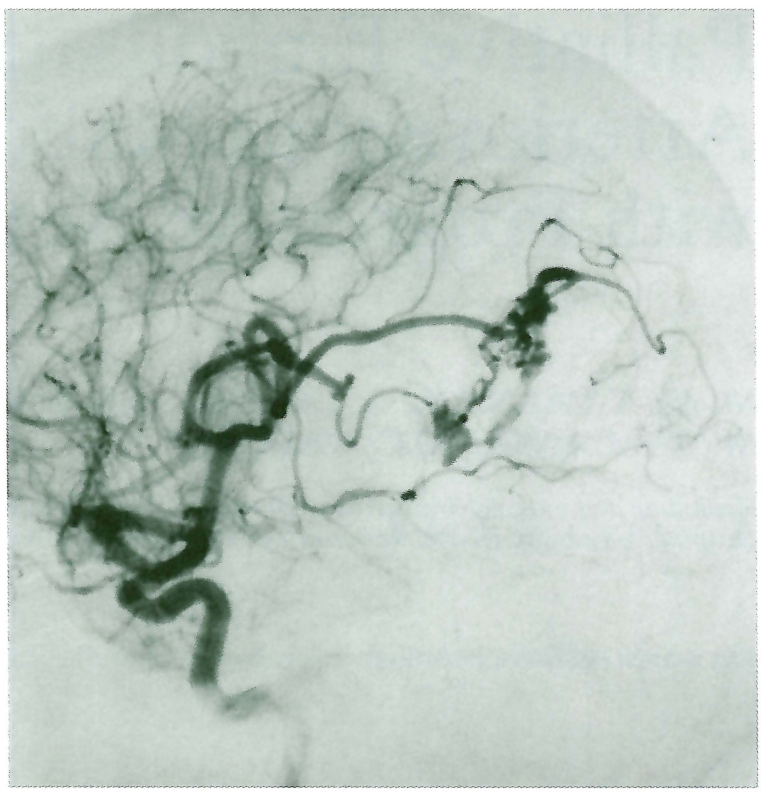

Figure 1

The patients were all conferenced in the presence of vascular neurosurgeons, interventional neuroradiologists and radiosurgery specialists and their AVMs were determined unfavorable for resection and not suitable for stereotactic radiosurgery.

Of the 24 patients initially referred to us because of a progressive neurological deficit subsequent careful documentation in our clinic did not confirm continued neurological worsening in seven patients and consequently these pa- 


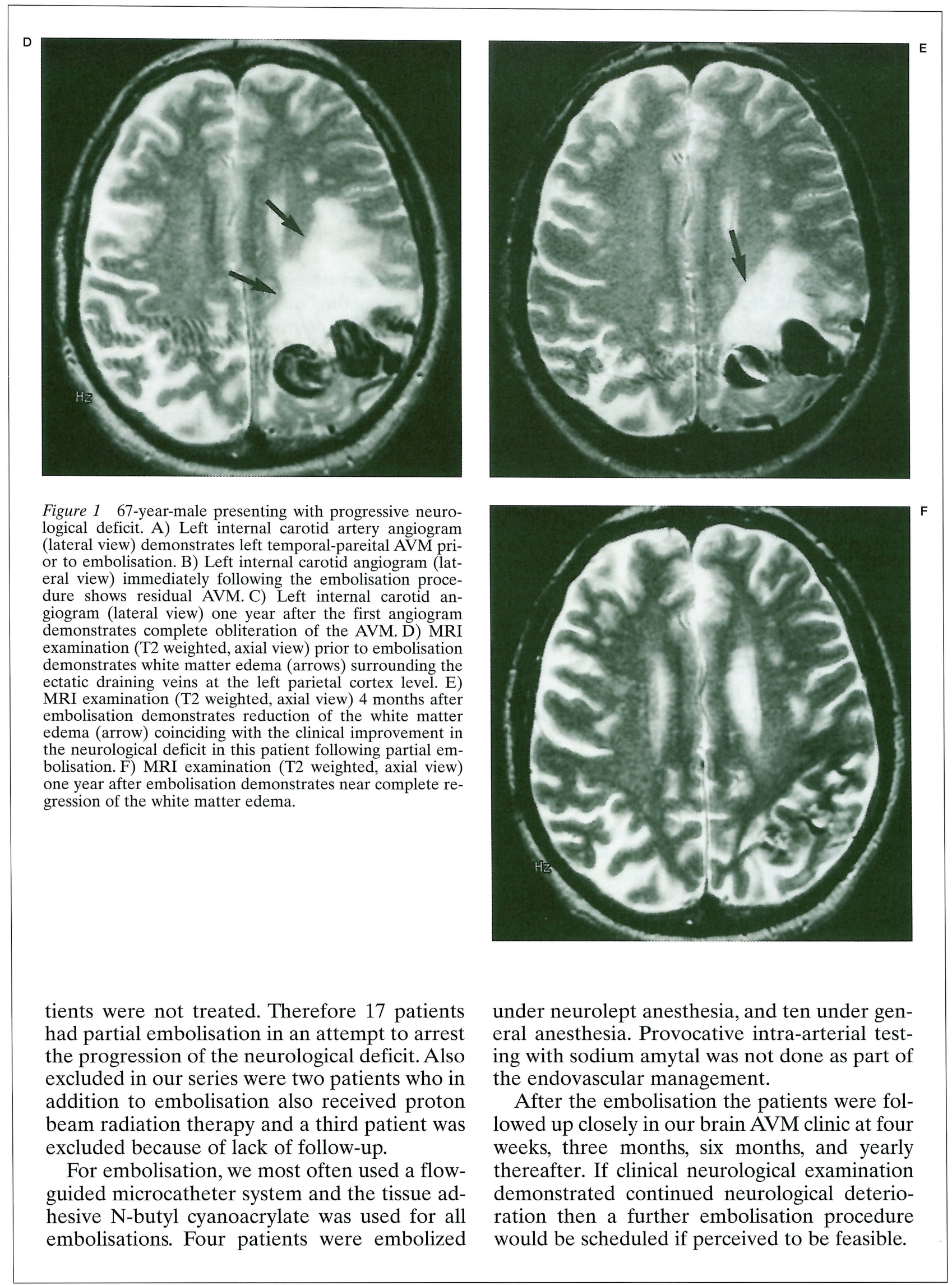




\section{Results}

\section{Study group}

We were able to partially embolize 17 brain AVMs in patients whose neurological symptoms were documented to be progressive. Subsequently two patients went on to receive proton beam radiation therapy following partial embolisation and one patient was lost to follow-up. These three patients were excluded from the study, leaving 14 patients for evaluation. The mean age of our patients was 35 years at the time of presentation (range 14-65 years). There were ten males and four females. Twelve patients had a single embolisation procedure, one patient had two procedures, and one had three procedures.

\section{Lesion location}

The AVM location was: thalamic/basal ganglia in seven, fronto-pareital in two, pareito-occipital in one, perisylvian in one, brainstem in two and cerebellum in two.

\section{Initial presentation}

Brainstem symptoms such as dysarthria, dysphagia, internuclear ophthalmoplegia, hearing loss, ataxia, nystagmus, or long tract signs were noted in six of 14 patients. Eight patients had progressive hemiparesis, two of whom originally presented with monoparesis of one upper limb, and all of these developed facial palsy. Pure hemisensory deficit followed by progressive motor deficit was seen in two patients. Focal and secondary generalized seizures were the initial presentation in two patients, both of whom subsequently developed progressive hemiparesis and expressive dysphasia. One patient presented with downward herniation of the hindbrain as a result of a large thalamic AVM draining into the posterior fossa veins. None of our patients presented with a haemorrhage but one patient died of a large brainstem bleed on follow-up.

\section{Pre and post embolisation follow-up.}

The mean duration of pre-embolisation assessment was 27 months (range two months to six years).Clinical deterioration in the form of progressive motor deficit involving limbs and/or motor cranial nerves was well documented by the referring physician and confirmed by our group in the AVM clinic. The mean duration of deterioration was 12.3 months (range 2 to 36 months) and the more rapid deterioration correlated with the severity of the deficit. In certain cases the delay in treatment was caused by patients' hesitation to accept our recommendation to treat. Our post embolisation mean follow-up duration was 29 months (range 2 to 60 months).

\section{Outcome}

We graded outcome as: improved, stable or worse depending on the patient's response with respect to the neurological status prior to the embolisation.

In this small series six patients $(42 \%)$ had clinical improvement in their neurological status following partial embolisation therapy. Stabilization of the neurological deficit, was achieved in seven patients $(50 \%)$, one of whom stabilized for two months but then worsened again over a two month period and finally died from a brainstem haemorrhage. One patient who had clinical improvement developed progressive thrombosis of the AVM after embolisation (figure 1).

Two complications occurred related to the embolisation in 14 patients. A 14-year-boy developed a subarachnoid haemorrhage following perforation of a tortuous lenticulostriate artery feeder while using an over the wire microcatheter system. The patient required admission to the intensive care unit for one week, but recovered completely to his premorbid status. The second complication occurred in a patient who was treated for a progressive right hemiparesis and progressive personality disorder. A flow directed microcatheter was glued into one of the feeders of his basal ganglia AVM. Upon withdrawal of the microcatheter this device broke and the distal portion became lodged in the M1 segment of the middle cerebral artery. He developed a sudden worsening of his neurological deficit but over the next 12 months he improved to a level well above his baseline status at presentation.

\section{Discussion}

From a surgical point of view difficult to treat intracranial arteriovenous malformations are those graded IV and V in the Spetzler and Martin grading system ${ }^{6}$. Unlike smaller lesions these AVMs don't usually present with in- 

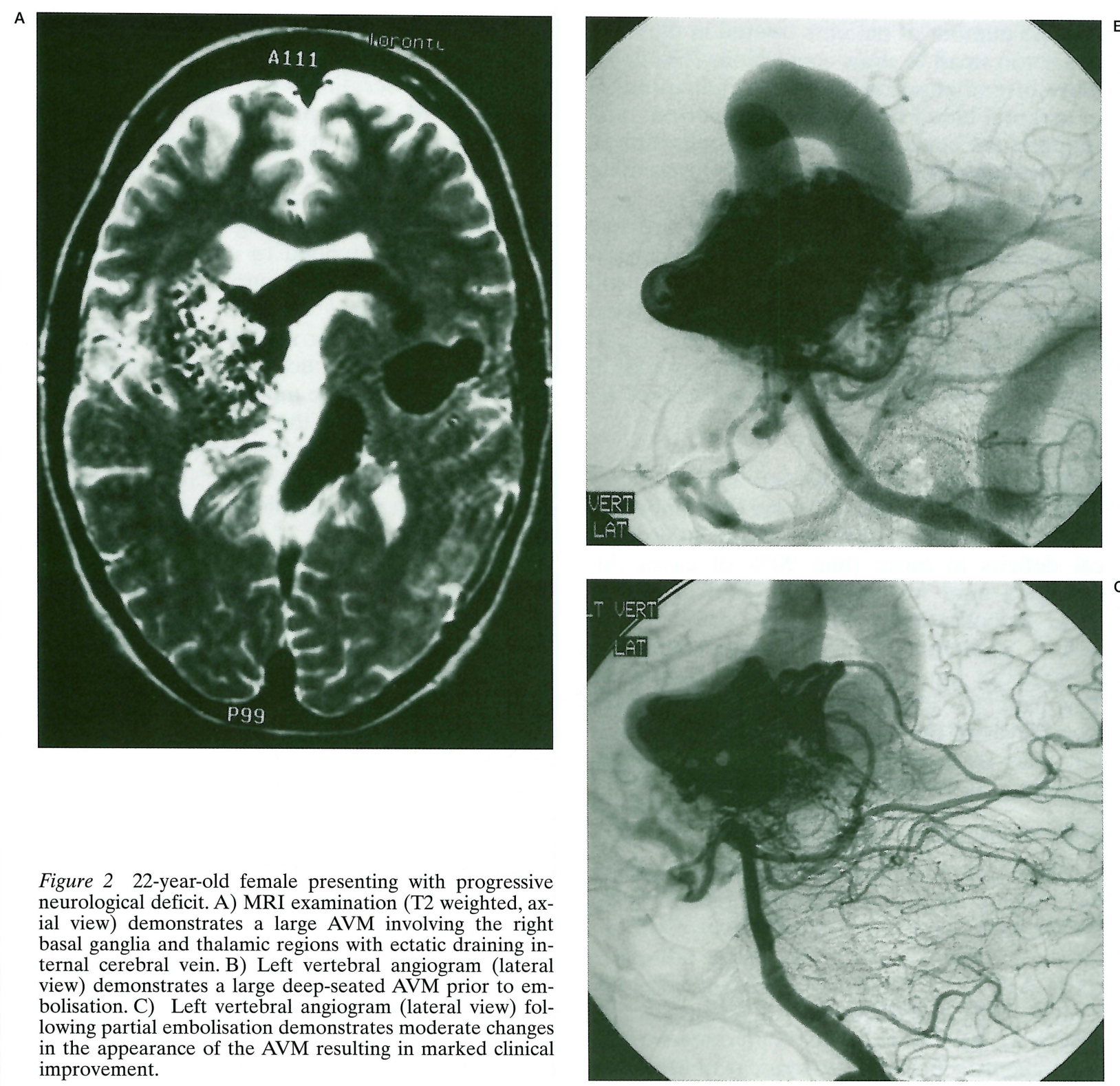

Figure 2 22-year-old female presenting with progressive neurological deficit. A) MRI examination (T2 weighted, axial view) demonstrates a large AVM involving the right basal ganglia and thalamic regions with ectatic draining internal cerebral vein. B) Left vertebral angiogram (lateral view) demonstrates a large deep-seated AVM prior to embolisation. C) Left vertebral angiogram (lateral view) following partial embolisation demonstrates moderate changes in the appearance of the AVM resulting in marked clinical improvement.

tracranial haemorrhage but rather with seizures or progressive neurological deficit possibly related to a steal phenomenon or venous congestion which in turn produces reduction in cerebral perfusion of the adjacent brain parenchyma ${ }^{4,5,7}$. Objective demonstration of these factors is often not possible, particularly in the deep-seated large AVMs, but increasing surrounding edema, possibly related to venous ischemia, was sometimes noted in our patients with large lobar AVMs in eloquent locations coinciding with the progression of the neuro-

logical deficit with reversal after successful embolisation treatment (figure 1).

A major management problem occurs when patients with large, inoperable AVMs begin to suffer from a progressive neurological deficit. Embolisation followed by radiosurgery may only lead to a cure if the nidus of the AVM can be reduced to a size that can be effectively treated with radiosurgery, which is usually $<3$ $\mathrm{cm}$ in the widest diameter ${ }^{8}$. Partial embolisation of so-called incurable AVMs is alluded to in the literature ${ }^{9,10,11,12,13,14}$ with conflicting results 
and the number of patients treated in these series is too small to draw conclusions. The capability of embolisation to induce redistribution of cerebral blood flow has been reported ${ }^{7}$, and supports the concept of partial embolisation in the management of brain AVMs presenting with progressive neurological deficits.

In our 14 patients treated for progressive neurological deficits we achieved clinical improvement in $42 \%$, stabilization in $50 \%$ and continued worsening in $7 \%$ of patients. Clinical stabilization and / or improvement did not always correspond to detectable angiographical changes in the large basal ganglia-thalamic lesions (figure 2).

Although the size of this series does not allow statistical analysis, we found that embolisation arrested clinical progression of neurological deficits in more than $90 \%$ of cases. Although a cure was never the goal in any of these embolisations, one of the patients who had a moderate clinical improvement did progress to an angiographic cure (figure 1), This can be explained by the progressive thrombosis of the AVM nidus which can occur following embolisation of the main AVM feeders with good intranidal penetration ${ }^{15}$.

\section{Embolisation Material and Strategy}

Many different types of embolic materials have been used for embolisation of brain AVMs, such as polyvinyl alcohol foam ${ }^{16}$, silk sutures ${ }^{17,18}$ ethanol ${ }^{19}$, coils, and tissue adhesives (NBCA) ${ }^{11,15,20,21,22}$. We have been using tissue adhesives (IBCA, NBCA) for 15 years and, like others, we have found it to be an effective embolic agent to produce permanent obliteration of brain AVMs at an acceptable complication rate $^{11,15,20,21,22}$.

Since embolisation is carried out as a unique treatment, the embolic agent must be permanent and reach the nidus, which excludes particulate matter and coils ${ }^{16}$ and be performed with acceptable risk, which presently excludes the use of alcohol ${ }^{19}$.

We prefer for the purpose of brain AVM embolisation the use of flow guided microcatheter systems, but superselective catheterization of perforating vessels may from time to time require the use of an over the wire system, with its inherent increased risk for complications. We tended to close off the largest shunts within the nidus first in order to achieve a significant heamodynamic impact. Great care was taken to scrutinize the angioarchitecture of the AVM nidus for possible weaknesses such as aneurysms in order to avoid redirection of bloodflow towards such perceived weaknesses ${ }^{23,24}$. If intranidal aneurysms were identified, then their feeding vessels were embolized first. We tended to embolize three or four of the largest AV shunts within the nidus during each session.

We embolised four AVMs under neurolept anesthesia and ten under general anesthesia. We have found it to be more convenient for the patient as well as for the interventional therapist to perform these procedures under general anesthesia, as it facilitates safe super-selective catheterization and embolisation of small feeders to the nidus.

We were able to perform these embolisations with a low morbidity of $7 \%$ non-permanent neurological deficit and zero mortality in the absence of physiological monitoring or provocative testing. Accordingly, all our brain AVM embolisations are currently carried out under general anesthesia and provocative testing was abandoned in 1986.

While partial embolisation appears to have a definite role in the management of patients presenting with progressive neurological deficit caused by large brain AVMs in eloquent locations, such embolisations did not appear to have an impact on the risk of future haemorrhage in these patients. In our overall experience with brain AVMs there proved to be a slightly increased risk for future haemorrhage in patients with larger AVMs in deep locations, irrespective of their initial presentation. It would therefore not be unexpected for AVMs in those locations to subsequently present with haemorrhage (as occurred in one of our 14 patients) since the embolisation was not targeted towards that goal but rather focussed on having a haemodynamic impact.

\section{Conclusions}

Palliative embolisation of large size brain AVMs in eloquent locations in patients presenting with progressive neurological deficit frequently results in clinical improvement or stabilisation of the deficit and has an acceptable morbidity. 


\section{References}

1 Richling B, Bavinzski G: Arterio-venous malformations of the basal ganglia. Surgical versus endovascular treatment. Acta Neurochir Supple 53: 50-59, 1991.

2 Tew JM, Lewis AI, Reichert KW: Management strategies and surgical techniques for deep-seated supratentorial arteriovenous malformations. Neurosurgery 36: 1065-1072, 1995.

3 Yamada S: Surgical approach to arteriovenous malformations in functional areas of the brain. In: Yamada S, (ed): Arteriovenous malformations in functional areas of the brain. Futura, Armonk, NY 1999: 1-123.

4 Brown RD, Wiebers DO et Al: The natural history of unruptured intracranial arteriovenous malformations. J Neurosurg 68: 352-357, 1988

5 Ondra SL, Troupp $\mathrm{H}$ et Al: The natural history of symptomatic arteriovenous malformation of the brain: a 24-year follow-up assessment. J Neurosurg 73: 387391, 1990.

6 Spetzler RF, Martin NA: A proposed grading system for arteriovenous malformations. J Neurosurg 65: 476483, 1986.

7 Hurst RW, Hackney DB et Al: Reversible arteriovenous malformation - induced venous hypertension as a cause of neurological deficits. Neurosurgery 30: 422425, 1992.

8 Gobin YP, Laurent A et Al: Treatment of brain arteriovenous malformation by embolization and radiosurgery. J Neurosurg 85: 19-28, 1996.

9 Benati A: Interventional neuroradiology for the treatment of inaccessible arterio-venous malformations. Acta Neurochir (wien) 118: 76-79, 1992.

10 Fox AJ, Girvin JP et Al: Rolandic Arteriovenous Malformations: Improvement in limb function by IBC embolization. Am J Neuroradiol 6: 575-582, 1985.

11 Hurst RW, Berenstein A et Al: Deep central arteriovenous malformations of the brain: the role of endovascular treatment . J Neurosurg 82: 190-195, 1995.

12 Sasaki T, Kurita $\mathrm{H}$ et Al: Arteriovenous malformations in the basal ganglia and thalamus: management and results in 101 cases. J Neurosurg 88: 285-292, 1998.

13 Sugita M, Takahashi A, Ogawa A: Improvement of cerebral blood flow and clinical symptoms associated with embolization of a large arteriovenous malformation: Case report. Neurosurgery 33: 748-752, 1993.

14 Zhengsong H, Qinshun D, Jingxian S: Percutaneous endovascular embolization of intracerebral arteriovenous malformations. Chinese Medical Journal 108: 413-419, 1995.

15 Debrun GM, Aletich V et Al: Embolization of the nidus of brain arteriovenous malformations with $\mathrm{n}$ butyl cyanoacrylate. Neurosurgery 40: 112-120, 1997.

16 Germano IM, Davis RL et Al: Histopathological follow-up study of 66 cerebral arteriovenous malformations after therapeutic embolization with polyvinyl alcohol. J Neurosurg 76: 607-614, 1992.

17 Deveikis JP, Manz HJ et Al: A clinical and neuropathologic study of silk suture as an embolic agent for brain arteriovenous malformations. Am J Neuroradiol 15: 263-271, 1994

18 Schmutz F, McAuliffe W et Al: Embolization of cerebral arteriovenous malformations with silk: Histopathologic changes and hemorrhagic complications. Am J Neuroradiol 18: 1233-1237, 1997.

19 Yakes WF, Krauth L et Al: Ethanol endovascular management of brain arteriovenous malformations: Initial results. Neurosurgery 40: 1145-1154, 1997.
20 Fournier D, TerBrugge KG et Al: Endovascular treatment of intracerebral arteriovenous malformations: experience in 49 cases. J Neurosurg 75: 228-233, 1991.

21 Valavanis A, Yasargil MG: The endovascular treatment of brain arteriovenous malformations. Adv Tech Stand Neurosurg 24: 131-214, 1998.

22 Wikholm G: Occlusion of cerebral arteriovenous malformations with N-butyl cyano-acrylate is permanent. Am J Neuroradiol 16: 479-482, 1995.

23 Abe T, Nemoto S et Al: Rupture of a cerebral aneurysm during embolization for a cerebral arteriovenous malformation. Am J Neuroradiol 16: 1818-1820, 1995.

24 Redekop G, TerBrugge KG et Al: Arterial aneurysms associated with cerebral arteriovenous malformations: classification, incidence, and risk of hemorrhage. J Neurosurg 89: 539-546, 1998.

Karel G. terBrugge MD. FRCP(C)

Professor of Radiology and Surgery

University of Toronto

Head Division of Interventional

and Diagnostic Neuroradiology

The Western Hospital

University Health Network.

399 Bathurst Street, Toronto, Ontario, Canada. M5T 2S8

E-mail: karel.terbrugge@uhn.on.ca 